\title{
Maximal Regularity for Stochastic Integral Equations
}

Wolfgang Desch, Stig-Olof Londen 
Maximal Regularity for Stochastic Integral Equations

\author{
Wolfgang Desch, Stig-Olof Londen
}




\section{Contact information}

Wolfgang Desch

Institut für Mathematik und Wissenschaftliches Rechnen

Karl-Franzens-Universität Graz

Heinrichstrasse 36

8010 Graz

Austria

georg.desch@uni-graz.at

Stig-Olof Londen

Aalto University

Department of Mathematics and Systems Analysis

PO Box 11100

FI-00076 Aalto

Finland

stig-olof.londen@tkk.fi

Aalto University publication series

SCIENCE + TECHNOLOGY 1/2011

Manuscript received 2011-01-07

(c) Author

ISBN 978-952-60-4021-9 (pdf)

ISSN-L 1799-4896

ISSN 1799-490X (pdf)

Aalto Print

Helsinki 2011

The publication can be read at http://math.tkk.fi/reports 
Author

Wolfgang Desch, Stig-Olof Londen

Name of the publication

Maximal regularity for stochastic integral equations

Publisher School of Science

Unit Department of Mathematics and Systems Analysis

Series Aalto University publication series SCIENCE + TECHNOLOGY 1/ 2011

Field of research

\section{Abstract}

AMS subject classifications: 60H15, 60H20, 45N05

We examine the stochastic parabolic integral equation of convolution type

$$
U(t)+A \int_{0}^{t} k_{1}(t-s) U(s) d s=\int_{0}^{t} k_{2}(t-s) G(s) d W_{H}(s), t \geq 0,
$$

where $U(t)$ takes values in $L_{q}(\mathcal{O} ; \mathbb{R})$ with $\mathcal{O}$ a $\sigma$-finite measure space, and $q \in[2, \infty)$. The linear operator $A$ maps $\mathcal{D}(A) \subset L_{q}(\mathcal{O} ; \mathbb{R})$ into $L_{q}(\mathcal{O} ; \mathbb{R})$, is nonnegative and admits a bounded $\mathbf{H}^{\infty}$-calculus on $L_{q}(\mathcal{O} ; \mathbb{R})$. The kernels are powers of $t$, with $k_{1}(t)=\frac{1}{\Gamma(\alpha)} t^{\alpha-1}, k_{2}(t)=\frac{1}{\Gamma(\beta)} t^{\beta-1}$, and $\alpha \in(0,2)$, $\beta \in\left(\frac{1}{2}, 2\right)$.

We show that, in the maximal regularity case, where

$$
\beta-\alpha \theta-\eta=\frac{1}{2}
$$

one has the estimate

$$
\left\|A^{\theta} D_{t}^{\eta} U\right\|_{L_{p}\left(\mathbb{R}^{+} \times \Omega ; L_{q}(\mathcal{O} ; \mathbb{R})\right)} \leq c\|G\|_{L_{p}\left(\mathbb{R}^{+} \times \Omega ; L_{q}(\mathcal{O} ; H)\right)},
$$

where $c$ is independent of $G$.

Here $D_{t}^{\eta}$ denotes fractional integration if $\eta \in(-1,0)$, and fractional differentiation if $\eta \in(0,1)$, both with respect to the $t$-variable.

The proof relies on recent work on stochastic differential equations by $\mathrm{v}$. Neerven, Veraar and Weis, and extends their maximal regularity result to the integral equation case.

Keywords stochastic integral equations, maximal regularity, bounded $\mathrm{H}$-infinity calculus, Volterra equations, singular kernels

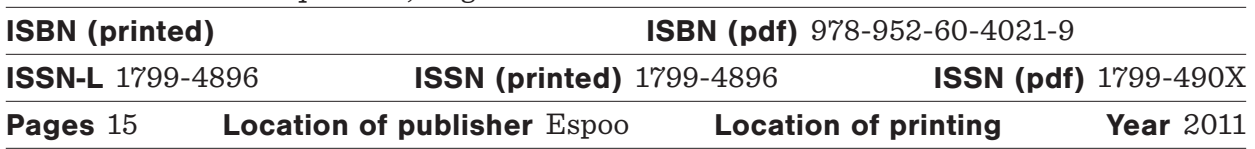




\title{
MAXIMAL REGULARITY FOR STOCHASTIC INTEGRAL EQUATIONS
}

\author{
W. Desch and S-O. Londen
}

Abstract We examine the stochastic parabolic integral equation of convolution type

$$
U(t)+A \int_{0}^{t} k_{1}(t-s) U(s) d s=\int_{0}^{t} k_{2}(t-s) G(s) d W_{H}(s), t \geq 0,
$$

where $U(t)$ takes values in $L_{q}(\mathcal{O} ; \mathbb{R})$ with $\mathcal{O}$ a $\sigma$-finite measure space, and $q \in[2, \infty)$. The linear operator $A$ maps $\mathcal{D}(A) \subset L_{q}(\mathcal{O} ; \mathbb{R})$ into $L_{q}(\mathcal{O} ; \mathbb{R})$, is nonnegative and admits a bounded $\mathbf{H}^{\infty}$-calculus on $L_{q}(\mathcal{O} ; \mathbb{R})$. The kernels are powers of $t$, with $k_{1}(t)=\frac{1}{\Gamma(\alpha)} t^{\alpha-1}, k_{2}(t)=\frac{1}{\Gamma(\beta)} t^{\beta-1}$, and $\alpha \in(0,2)$, $\beta \in\left(\frac{1}{2}, 2\right)$.

We show that, in the maximal regularity case, where

$$
\beta-\alpha \theta-\eta=\frac{1}{2}
$$

one has the estimate

$$
\left\|A^{\theta} D_{t}^{\eta} U\right\|_{L_{p}\left(\mathbb{R}^{+} \times \Omega ; L_{q}(\mathcal{O} ; \mathbb{R})\right)} \leq c\|G\|_{L_{p}\left(\mathbb{R}^{+} \times \Omega ; L_{q}(\mathcal{O} ; H)\right)},
$$

where $c$ is independent of $G$.

Here $D_{t}^{\eta}$ denotes fractional integration if $\eta \in(-1,0)$, and fractional differentiation if $\eta \in(0,1)$, both with respect to the $t$-variable.

The proof relies on recent work on stochastic differential equations by $\mathrm{v}$. Neerven, Veraar and Weis, and extends their maximal regularity result to the integral equation case.

\section{INTRODUCTION}

We investigate the stochastic integral equation

$$
U(t)+A \int_{0}^{t} k_{1}(t-s) U(s) d s=\int_{0}^{t} k_{2}(t-s) G(s) d W_{H}(s), t \geq 0
$$

where we require $U(t)$ to take values in $L_{q}(\mathcal{O} ; \mathbb{R})$ almost surely, with $\mathcal{O}$ a $\sigma$-finite measure space, and $q \in[2, \infty)$. The linear operator $A$ maps $\mathcal{D}(A) \subset L_{q}(\mathcal{O} ; \mathbb{R})$ into $L_{q}(\mathcal{O} ; \mathbb{R})$, is nonnegative and admits a bounded $\mathbf{H}^{\infty}$-calculus on $L_{q}(\mathcal{O} ; \mathbb{R})$. The kernels are powers of $t$, with $k_{1}(t)=\frac{1}{\Gamma(\alpha)} t^{\alpha-1}, k_{2}(t)=\frac{1}{\Gamma(\beta)} t^{\beta-1}$, and $\alpha \in(0,2), \beta \in\left(\frac{1}{2}, 2\right)$. The integral on the left side of (1.1) is a standard Bochner integral.

Let $(\Omega, \mathcal{A}, \mathcal{P})$ be a probability space with the filtration $\mathcal{F}=\left(\mathcal{F}_{t}\right)_{t \geq 0}$, and take $W_{H}(t), t \geq 0$, to be a cylindrical $\mathcal{F}$-Brownian motion on the separable Hilbert space $H$. The stochastic integral with respect to $W_{H}$ on the right side of $(1.1)$ is an $L_{q}(\mathcal{O} ; \mathbb{R})$-valued random variable as 
defined, e.g., in [7, Sec. 2.1]. Assume $G \in L_{p}\left(\mathbb{R}^{+} \times \Omega ; L_{q}(\mathcal{O} ; H)\right)$ for some $p \in(2, \infty)$ and that $G$ is $\mathcal{F}$-adapted.

Define the stochastic convolution process $U(t)$ by

$$
U(t)=\int_{0}^{t} S_{\alpha \beta}(t-s) G(s) d W_{H}(s), t \geq 0 .
$$

Here $S_{\alpha \beta}(t) \in \mathcal{L}\left(L_{q}(\mathcal{O} ; \mathbb{R})\right), t \geq 0$, is the resolvent (see Section 2 below) associated with $A, k_{1}$, and $k_{2}$, i.e., the solution of

$$
S_{\alpha \beta}+A\left(k_{1} * S_{\alpha \beta}\right)=k_{2} I .
$$

The process $U(t)$ solves (1.1), at least formally.

Let $D_{t}^{\eta}$ denote fractional integration (if $\eta \in(-1,0)$ ) and fractional differentiation (if $\eta \in(0,1)$ ); both with respect to the $t$-variable. For $\theta \in(0,1)$, let $A^{\theta}$ stand for the fractional power of $A$.

Our main result concerns the maximal regularity case

$$
\beta-\alpha \theta-\eta=\frac{1}{2} \text {. }
$$

We show, assuming this and making an appropriate spectral assumption on $A$, that $A^{\theta} D_{t}^{\eta} U$ is welldefined and satisfies

$$
\left\|A^{\theta} D_{t}^{\eta} U\right\|_{L_{p}\left(\mathbb{R}^{+} \times \Omega ; L_{q}(\mathcal{O} ; \mathbb{R})\right)} \leq c\|G\|_{L_{p}\left(\mathbb{R}^{+} \times \Omega ; L_{q}(\mathcal{O} ; H)\right)},
$$

with the constant $c$ independent of $G$, but depending on $p, q, \alpha, \beta, \eta, \theta$. If $q=2$, the estimate (1.4) also holds for $p=2$.

Essentially the same equation as (1.1) has previously been considered in [4] under the stronger condition

$$
\beta-\alpha \theta-\eta>\frac{1}{2}
$$

with $q=p \in[2, \infty)$ and $t$ in compact sets . However, the assumption that $A$ admits a bounded functional calculus was not made in [4], only the assumption that $A$ be nonnegative, including the appropriate spectral condition. Estimates analogous to (1.4) were obtained.

Technically the proofs of [4] rely on an approach due to Krylov making use of the Burkholder-Davis-Gundy inequality and developed for stochastic differential equations, i.e., for the case $\alpha=\beta=1$ in (1.1), and with $-A$ a second order elliptic differential operator on $\mathbb{R}^{n}$. In this case, Krylov's approach can be used to obtain maximal regularity, however, it relies on a deep Paley-Littlewood inequality [5], [6]. For general sectorial $A$ in the integral equation case (i.e., $\alpha \in(0,2), \beta \in\left(\frac{1}{2}, 2\right)$ ), this inequality may be replaced by estimates on the Dunford integral - but then an infinitesimal loss of regularity must be allowed, as illustrated by (1.5). 
To obtain maximal regularity in the integral equation case - which in [4] was done only for the Laplacian on $\mathbb{R}^{n}$ - a highly nontrivial generalization of Krylov's lemma was required [3].

Our result in this paper relies strongly on the deep approach of the recent paper [7], concerned with maximal regularity of (1.1) in the differential equation case, i.e., $\alpha=\beta=1$. In fact, the major part of the proof of [7] can be adapted almost without changes to apply to (1.1). The only necessary major alteration is the construction of new, different $k_{j}$-functions (see Proposition 3.3 below). Note that our $k_{j}$ functions are constructed by a different approach and do not reduce to those of [7] in case $\alpha=\beta=1$.

As already stated, our main result (Theorem 3.1) gives the estimate (1.4). Observe that this estimate may be interpreted as an estimate on $D_{t}^{\eta}\left(A^{\theta} U\right)$, i.e., on the fractional time-derivative of $A^{\theta} U$. Hence this estimate combined with the additional assumption $0 \in \rho(A)$ directly gives maximal space-time $L_{p}$-regularity, i.e., maximal regularity in Bessel potential spaces. (See Corollary 3.2 below). Therefore, no modification or extension of the Da Prato-Kwapien-Zabczyk factorization argument used in [7] to obtain Bessel potential space regularity is required here. Obviously, this simpler proof also applies in case $\alpha=\beta=1$.

In Section 2 we formulate some technical preliminaries. Our results are given in Section 3. In Section 4 we show how Corollary 3.2 follows from Theorem 3.1. Section 5 is devoted to the construction of $k_{j}$ functions representing the resolvent. Section 6 contains the proof of Theorem 3.1.

\section{TEChNiCAL PRELIMinaries}

First, we consider fractional integration and differentiation in time.

Definition 2.1. Let $X$ be a Banach space and $\eta \in(0,1)$, let $u \in$ $L_{1}((0, T) ; X)$ for some $T>0$.

1) Fractional integration in time is defined by

$$
D_{t}^{-\eta} u=\frac{1}{\Gamma(\eta)} t^{\eta-1} * u
$$

2) We say that $u$ has a fractional derivative of order $\eta>0$ provided $u=D_{t}^{-\eta} f$, for some $f \in L_{1}((0, T) ; X)$. If this is the case, we write $D_{t}^{\eta} u=f$.

Obviously, $D_{t}^{0} f=f$

For the equivalence of fractional derivatives in $L_{p}$ and fractional powers of the realization of the derivative in $L_{p}$ we have:

Lemma 2.2. [2, Proposition 2] Let $p \in[1, \infty), X$ a Banach space and define

$$
\mathcal{D}(L)=\left\{u \in W_{p}^{1}((0, T) ; X) \mid u(0)=0\right\}, L u=u^{\prime}, u \in \mathcal{D}(L) .
$$


Then, with $\beta \in(0,1)$

$$
L^{\beta} u=D_{t}^{\beta} u, \quad u \in \mathcal{D}\left(L^{\beta}\right),
$$

where $\mathcal{D}\left(L^{\beta}\right)$ coincides with the set of functions $u$ having a fractional derivative of order $\beta$ in $L_{p}$, i.e.,

$$
\mathcal{D}\left(L^{\beta}\right)=\left\{u \in L_{p}((0, T) ; X) \mid t^{-\beta} * u \in W_{0, p}^{1}((0, T) ; X)\right\} .
$$

In particular, $D_{t}^{\beta}$ is closed.

We then recall the definition of the resolvent associated with $A, k_{1}$, and $k_{2}$.

Definition 2.3. Let $X$ be a Banach space, let $A$ map $\mathcal{D}(A) \subset X \rightarrow$ $X$ and let $A$ be sectorial. Let $k_{1}, k_{2}$ be power functions as in the introduction. Let $\phi_{A}$ be the spectral angle of $A$ and assume

$$
\phi_{A}<\pi\left(1-\frac{\alpha}{2}\right) .
$$

The resolvent $S_{\alpha \beta}$ associated with $A, k_{1}$ and $k_{2}$ is defined by

$$
S_{\alpha \beta}(t) v=(2 \pi i)^{-1} \int_{\Gamma_{1, \psi}} e^{\lambda t}\left(\lambda^{\alpha} I+A\right)^{-1} \lambda^{\alpha-\beta} v d \lambda, t>0,
$$

for $v \in X$; and where $\psi \in\left(\frac{\pi}{2}, \min \left\{\pi, \frac{\pi-\phi_{A}}{\alpha}\right\}\right)$, and

$$
\Gamma_{r, \psi}=\left\{r e^{i t}|| t \mid \leq \psi\right\} \cup\left\{\rho e^{i \psi} \mid r<\rho<\infty\right\} \cup\left\{\rho e^{-i \psi} \mid r<\rho<\infty\right\} .
$$

As to the properties of $S_{\alpha \beta}$ we have

Remark 2.4. [4] Let $\alpha \in(0,2), \beta \in\left(\frac{1}{2}, 2\right), \theta \in[0,1], \eta \in(-1,1)$. Let $S_{\alpha \beta}(t)$ be the resolvent of Definition 2.3. Then one has

$$
\begin{aligned}
& S_{\alpha \beta}(t) \in \mathcal{L}(X), t>0 ; \quad S_{\alpha \beta}(t) v \in \mathcal{D}(A), t>0, v \in X, \\
& \sup _{t>0}\left\|t^{1+\alpha \theta-\beta+\eta} D_{t}^{\eta} A^{\theta} S_{\alpha \beta}(t)\right\|_{\mathcal{L}(X)}<\infty, \\
& S_{\alpha \beta}(t)+A \int_{0}^{t} k_{1}(t-s) S_{\alpha \beta}(s) d s=k_{2}(t) I, \quad t>0 .
\end{aligned}
$$

For the proof of these properties, see [4].

\section{Results}

Our main result is the following:

Theorem 3.1. Take $(\Omega, \mathcal{A}, \mathcal{P})$ to be a probability space with filtration $\mathcal{F}=\left\{\mathcal{F}_{t}\right\}_{t \geq 0}$. Let $W_{H}(t), t \geq 0$, be a cylindrical $\mathcal{F}$-Brownian motion on the Hilbert space $H$. Let $p \in[2, \infty)$. 
Let $\alpha \in(0,2), \beta \in\left(\frac{1}{2}, 2\right), \theta \in[0,1)$, and $\eta \in(-1,1)$, and suppose that

$$
\beta-\alpha \theta-\eta=\frac{1}{2}
$$

Let $q \in[2, \infty)$. Assume the operator $A$ admits a bounded $H^{\infty}$ calculus on $L_{q}(\mathcal{O} ; \mathbb{R})$ of angle $\omega$ satisfying

$$
\omega<\pi\left(1-\frac{\alpha}{2}\right)
$$

Let $S_{\alpha \beta}(t) \in \mathcal{L}\left(L_{q}(\mathcal{O} ; \mathbb{R})\right), t \geq 0$, be the resolvent associated with $A$, $k_{1}$, and $k_{2}$, as defined in Definition 2.3, (where $X=L_{q}(\mathcal{O} ; \mathbb{R})$ ).

Define, for any $\mathcal{F}$-adapted $G \in L_{p}\left(\mathbb{R}^{+} \times \Omega ; L_{q}(\mathcal{O} ; H)\right)$, the stochastic convolution $U(t)$ by

$$
U(t)=\int_{0}^{t} S_{\alpha \beta}(t-s) G(s) d W_{H}(s), t \geq 0 .
$$

Then $D_{t}^{\eta} U$ is welldefined in $L_{q}(\mathcal{O} ; \mathbb{R})$, takes values in the domain $\mathcal{D}\left(A^{\theta}\right)$ almost surely and satisfies, for $2<p<\infty$,

$$
\left\|A^{\theta} D_{t}^{\eta} U\right\|_{L_{p}\left(\mathbb{R}^{+} \times \Omega ; L_{q}(\mathcal{O} ; \mathbb{R})\right)} \leq c\|G\|_{L_{p}\left(\mathbb{R}^{+} \times \Omega ; L_{q}(\mathcal{O} ; H)\right)},
$$

with the constant $c$ independent of $G$. If $q=2$, then this maximal regularity holds also for $p=2$.

Note the necessary condition $\beta>\frac{1}{2}$. Thus, e.g., the case $\theta=0$, $\eta \leq 0$, is not covered by Theorem 3.1. See however, the comments following Corollary 3.2.

If $A$ is invertible, then we may, in the maximal regularity case, formulate a result in Bessel potential spaces.

Corollary 3.2. Let, in addition to the assumptions of Theorem 3.1, $0 \in \rho(A)$, and let $\eta \in[0,1)$ and assume

$$
\beta-\frac{1}{2}<\alpha
$$

Then

$$
\|U\|_{H_{p}^{\eta}\left(\mathbb{R}^{+} ; L_{p}\left(\Omega ; \mathcal{D}\left(A^{\theta}\right)\right)\right)} \leq c\|G\|_{L_{p}\left(\mathbb{R}^{+} \times \Omega ; L_{q}(\mathcal{O} ; H)\right)},
$$

with the constant $c$ independent of $G$.

Note that with $\alpha=\beta=1$ (the differential equation case of [7] ), the condition (3.4) is trivially satisfied.

Also note that in the differential equation case (3.5) yields

$$
\|U\|_{H^{\theta, p}\left(\mathbb{R}^{+} ; L_{p}\left(\Omega ; \mathcal{D}\left(A^{\frac{1}{2}-\theta}\right)\right)\right)} \leq c\|G\|_{L_{p}\left(\mathbb{R}^{+} \times \Omega ; L_{q}(\mathcal{O}: H)\right)},
$$

for $\theta \in\left[0, \frac{1}{2}\right]$. (Cf.[7, Theorem 1.2(i)]).

Suppose next that

$$
\beta-\alpha \theta-\eta>\frac{1}{2}
$$


In the case where $p=q$, and $\mathbb{R}^{+}$is replaced by compact timeintervals we may apply results of [4]. These results do not require that $A$ admits a bounded $H^{\infty}$-calculus but only that $A$ is sectorial and satisfies the spectral condition (2.1).

If (3.6) holds and we wish to consider $\mathbb{R}^{+}$, and with $p$ not necessarily equal to $q$, then we may, for certain parameter values, argue as follows.

Suppose there exists $\theta_{0} \in(\theta, 1)$ such that $\beta-\alpha \theta_{0}-\eta=\frac{1}{2}$. Then, by Theorem 3.1,

$$
\left\|A^{\theta_{0}} D_{t}^{\eta} U\right\|_{L_{p}\left(\mathbb{R}^{+} \times \Omega ; L_{q}(\mathcal{O} ; \mathbb{R})\right)} \leq c\|G\|_{L_{p}\left(\mathbb{R}^{+} \times \Omega ; L_{q}(\mathcal{O} ; H)\right)},
$$

with $c$ independent of $G$. Arguing as in the proof of Corollary 3.2 we may then proceed to obtain norm estimates on $U$ in Bessel potential spaces.

Finally observe that Theorem 3.1 and Corollary 3.2 allow us to interpolate analogously as in [7, Theorem $1.2(2)]$ to obtain maximal estimates.

To prove Theorem 3.1, we need Proposition 3.3 below. This proposition contains the essential new element needed for the appropriate extension of the results of [7].

Below, we let $H^{\infty}\left(\Sigma_{\sigma}\right)$ denote the Banach space of all bounded analytic functions $\varphi$ that map $\Sigma_{\sigma}=\{z \in \mathbb{C} \backslash 0|| \arg z \mid<\sigma\} \rightarrow \mathbb{C}$ with the supremum norm. The linear subspace of $H^{\infty}\left(\Sigma_{\sigma}\right)$ consisting of all functions $\varphi$ satisfying an estimate

$$
|\varphi(z)| \leq \frac{c|z|^{\epsilon}}{\left(1+|z|^{2}\right)^{\epsilon}}, z \in \Sigma_{\sigma}
$$

for some $\epsilon>0$, and some $c<\infty$, is denoted by $H_{0}^{\infty}\left(\Sigma_{\sigma}\right)$.

Proposition 3.3. Let $\alpha \in(0,2), \beta \in\left(\frac{1}{2}, 2\right), \theta \in[0,1)$ and $\eta \in(-1,1)$. Assume the operator $A$ admits a bounded $H^{\infty}$-calculus on the Banach space $X$ of angle $\omega$ satisfying

$$
\omega<\pi\left(1-\frac{\alpha}{2}\right)
$$

Let (3.1) be satisfied.

Let $S_{\alpha \beta}(t), t \geq 0$, be the resolvent associated with $A, \frac{1}{\Gamma(\alpha)} t^{\alpha-1}$, $\frac{1}{\Gamma(\beta)} t^{\beta-1}$, as defined in Definition 2.3.

Then there exists $\omega_{1} \in(\omega, \pi)$, and for $j= \pm 1$ functions $k_{j}:(0, \infty) \times$ $(0, \infty) \rightarrow \mathbb{C}$, and $\varphi_{j} \in H_{0}^{\infty}\left(\Sigma_{\omega_{1}}\right)$ such that, for $t>0, x \in X$,

$$
A^{\theta} D_{t}^{\eta} S_{\alpha \beta}(t) x=\sum_{j= \pm 1} \int_{0}^{\infty} k_{j}(u, t)\left[\varphi_{j}(u A)\right]^{2} x \frac{d u}{u} .
$$

Moreover, for fixed $u$ we have $k_{j}(u, \cdot) \in W_{1}^{1}((0, \infty) ; \mathbb{C})$, with

$$
\int_{0}^{\infty} t^{\frac{1}{2}}\left|\frac{\partial}{\partial t} k_{j}(u, t)\right| d t \leq M
$$


where the constant $M$ is independent of $u$, but depends on the parameters $\alpha, \beta, \theta, \eta$ and on the norm of the operator $H^{\infty}\left(\Sigma_{\omega_{1}}\right) \rightarrow \mathcal{L}(X)$ given by $\varphi \rightarrow \varphi(A)$.

Proposition 3.3 is proved in Section 5, via three lemmas.

In Section 6, we briefly outline the proof of Theorem 3.1, using the proof of [7], combined with Proposition 3.3 above. Our goal in this outline is only to demonstrate that Proposition 3.3, combined with the proof of [7] is sufficient to prove Theorem 3.1.

\section{Proof of Corollary 3.2}

Proof. First recall that by the assumption $0 \in \rho(A)$,

$$
\|U\|_{H_{p}^{\eta}\left(\mathbb{R}^{+} ; L_{p}\left(\Omega ; \mathcal{D}\left(A^{\theta}\right)\right)\right)}=\left\|A^{\theta} U\right\|_{H_{p}^{\eta}\left(\mathbb{R}^{+} ; L_{p}\left(\Omega ; L_{q}(\mathcal{O} ; \mathbb{R})\right)\right)} .
$$

Then note that (see $[9$, p.29, Example 2.8.1] and recall that $U(t=0)=$ $0)$ we may take

$$
\left\|A^{\theta} U\right\|_{H_{p}^{\eta}\left(\mathbb{R}^{+} ; L_{p}\left(\Omega ; L_{q}(\mathcal{O}: \mathbb{R})\right)\right)}=\left\|A^{\theta} D_{t}^{\eta} U\right\|_{L_{p}\left(\mathbb{R}^{+} \times \Omega ; L_{q}(\mathcal{O} ; \mathbb{R})\right)}+\left\|A^{\theta} u\right\|_{L_{p}\left(\mathbb{R}^{+} \times \Omega ; L_{q}(\mathcal{O} ; \mathbb{R})\right)} .
$$

By (3.3) of Theorem 3.1,

$$
\left\|A^{\theta} D_{t}^{\eta} U\right\|_{L_{p}\left(\mathbb{R}^{+} \times \Omega ; L_{q}(\mathcal{O} ; \mathbb{R})\right)} \leq c\|G\|_{L_{p}\left(\mathbb{R}^{+} \times \Omega ; L_{q}(\mathcal{O} ; H)\right)} .
$$

Then define $\theta_{0}$ by $\beta-\alpha \theta_{0}=\frac{1}{2}$. By the assumption $\beta>\frac{1}{2}$ we have $\theta_{0}>0$. By (3.4), $\theta_{0}<1$. Then, by Theorem 3.1,

$$
\left\|A^{\theta_{0}} U\right\|_{L_{p}\left(\mathbb{R}^{+} \times \Omega ; L_{q}(\mathcal{O} ; \mathbb{R})\right)} \leq c\|G\|_{L_{p}\left(\mathbb{R}^{+} \times \Omega ; L_{q}(\mathcal{O} ; H)\right)} .
$$

By the assumption $0 \in \rho(A)$, and by the fact that $\theta_{0}>\theta$, we have that $\left(A^{\theta_{0}-\theta}\right)^{-1}$ exists. See, e.g. [1, Lemma 5.3.5]. Thus

$$
\left\|A^{\theta} u\right\|_{L_{p}\left(\mathbb{R}^{+} \times \Omega ; L_{q}(\mathcal{O} ; \mathbb{R})\right)} \leq c\|G\|_{L_{p}\left(\mathbb{R}^{+} \times \Omega ; L_{q}(\mathcal{O} ; H)\right)} .
$$

From (4.1) - (4.4) we have (3.5).

\section{Proof of Proposition 3.3}

Lemma 5.1. Let the assumptions of Proposition 3.3 hold. Choose $\rho, \omega_{1}$ such that

$$
\rho \in\left(\frac{\pi}{2}, \pi\right), \omega_{1} \in(\omega, \pi), \alpha \rho+\omega_{1}<\pi .
$$

By (3.7) this is possible. Then, for $j= \pm 1$,

a) The function $s \rightarrow\left(s e^{i j \rho}\right)^{\alpha}\left(\left(s e^{i j \rho}\right)^{\alpha}+A\right)^{-1}$ is welldefined and uniformly bounded in $\mathcal{L}(X)$ for $s \in(0, \infty)$.

b) Define, for $j= \pm 1$,

$$
\psi_{j}(z)=\frac{z^{\theta}}{1+e^{-i j \rho \alpha} z} .
$$


Then, for $j= \pm 1$, there exists a function $\varphi_{j} \in H_{0}^{\infty}\left(\Sigma_{\omega_{1}}\right)$ such that

$$
\psi_{j}(z)=\varphi_{j}(z)^{2}, z \in \Sigma_{\omega_{1}} .
$$

Proof. To prove (a), note first that for $s>0$, by (5.1),

$$
\mu=\left(s e^{i j \rho}\right)^{\alpha} \in \Sigma_{\pi-\omega_{1}} .
$$

Consequently,

$$
\sup _{z \in \Sigma_{\omega_{1}}}\left|\frac{\mu}{\mu+z}\right|=\sup _{z \in \Sigma_{\omega_{1}}}\left|\frac{1}{1+\frac{z}{\mu}}\right| \leq c
$$

with $c$ independent of $\mu$. Now note that $\omega_{1}>\omega$ and apply the bounded $H^{\infty}$-calculus of $A$ to get a).

To prove (b), take $z \in \Sigma_{\omega_{1}}$ and notice that by (5.1) there exists a constant $M$ such that

$$
\left|1+e^{-i j \rho \alpha} z\right| \geq M|z|, \quad z \in \Sigma_{\omega_{1}} .
$$

Thus, since $\theta<1, \psi_{j}(z) \in H_{0}^{\infty}\left(\Sigma_{\omega_{1}}\right)$. Write

$$
\psi_{j}(z)=\left[z^{-\theta}+e^{-i j \rho \alpha} z^{1-\theta}\right]^{-1}
$$

to see that for $z \in \Sigma_{\omega_{1}}$,

$$
\left|\arg \left(\psi_{j}(z)\right)\right| \leq \max \left(\omega_{1}, \rho \alpha+\omega_{1}\right)<\pi .
$$

Therefore, one may take $\phi_{j}=\left(\psi_{j}\right)^{\frac{1}{2}} \in H_{0}^{\infty}\left(\Sigma_{\omega_{1}}\right)$, where $z^{\frac{1}{2}}$ denotes any branch of the square root in the slotted plane $\mathbb{C} \backslash(-\infty, 0]$.

Lemma 5.2. Under the assumptions of Proposition 3.3, and with $\rho$ as in Lemma 5.1; define for $j= \pm 1 ; t, u>0$;

$$
k_{j}(u, t)=\frac{1}{2 \pi i \alpha} e^{i j \rho(1+\eta-\beta)} u^{-\frac{1}{2 \alpha}} e^{e^{i j \rho} t u^{-1 / \alpha}} .
$$

Then, for fixed $u>0$, we have $k_{j} \in W_{1}^{1}((0, \infty) ; \mathbb{C})$ and

$$
\int_{0}^{\infty} \sqrt{t}\left|\frac{\partial}{\partial t} k_{j}(u, t)\right| d t \leq M
$$

with a constant $M$ independent of $u$.

Proof. By the fact that $\rho>\frac{\pi}{2}$, we have $\Re\left(e^{i j \rho}\right)<0$, so that for fixed $u$ the function $k_{j}(u, \cdot) \in W_{1}^{1}((0, \infty) ; \mathbb{C})$. Estimating the integral gives (with suitable constants $K$ that may vary from line to line, and a change of variables $s=t u^{-\frac{1}{\alpha}}$ ), 


$$
\begin{aligned}
& \int_{0}^{\infty} \sqrt{t}\left|\frac{\partial}{\partial t} k_{j}(u, t)\right| d t \\
\leq & K \int_{0}^{\infty} \sqrt{t} u^{-1 /(2 \alpha)}\left|\frac{\partial}{\partial t} e^{e^{i j \rho} t u^{-1 / \alpha}}\right| d t \\
= & K \int_{0}^{\infty} \sqrt{t u^{-1 / \alpha}}\left|e^{i j \rho} u^{-1 / \alpha} e^{e^{i j \rho} t u^{-1 / \alpha}}\right| d t \\
= & K \int_{0}^{\infty} \sqrt{t u^{-1 / \alpha}}\left|e^{e^{i j \rho} t u^{-1 / \alpha}}\right| u^{-1 / \alpha} d t \\
= & K \int_{0}^{\infty} \sqrt{s}\left|e^{e^{i j \rho} s}\right| d s \\
= & K .
\end{aligned}
$$

Lemma 5.3. With the conditions of Proposition 3.3, with $\rho, \psi_{j}$ as in Lemma 5.1, and $k_{j}$ as in Lemma 5.2, we have for $t>0, x \in X$,

$$
A^{\theta} D_{t}^{\eta} S_{\alpha \beta}(t) x=\Sigma_{j= \pm 1} \int_{0}^{\infty} k_{j}(u, t) \psi_{j}(u A) x \frac{d u}{u} .
$$

Proof. The Laplace transform of $A^{\theta} D_{t}^{\eta} S_{\alpha \beta}(t)$ is given by (see [4, Definition 5.4],

$$
\lambda^{\eta+\alpha-\beta} A^{\theta}\left(\lambda^{\alpha} I+A\right)^{-1} .
$$

We let, for $r>0, \Gamma_{r}$ be the contour

$$
\begin{aligned}
& e^{-i \rho}|t|, \quad t \leq-r, \\
& e^{i \rho} t, \quad t \geq r \\
& e^{i s} r, \quad|s| \leq \rho .
\end{aligned}
$$

Then

$$
A^{\theta} D_{t}^{\eta} S_{\alpha \beta}(t) x=\frac{1}{2 \pi i} \int_{\Gamma_{r}} e^{\lambda t} \lambda^{\eta+\alpha-\beta} A^{\theta}\left(\lambda^{\alpha} I+A\right)^{-1} x d \lambda .
$$

Note that

$$
\sup _{\lambda \in \Gamma_{r}}\left\|A^{\theta} \lambda^{\alpha(1-\theta)}\left(\lambda^{\alpha} I+A\right)^{-1}\right\|_{\mathcal{L}(X)}<\infty
$$

and so, by (3.1),

$$
\left\|\int_{\left\{e^{i s} r|| s \mid \leq \rho\right\}} e^{\lambda t} \lambda^{\eta+\alpha-\beta} A^{\theta}\left(\lambda^{\alpha} I+A\right)^{-1} x d \lambda\right\|_{X} \leq K r^{\frac{1}{2}},
$$

for some constant $K$ independent of $r$. Consequently, by analyticity we may without loss of generality take $r=0$. Write $\Gamma=\Gamma_{0}$. We then have, for $t>0$, 


$$
\begin{aligned}
& D_{t}^{\eta} A^{\theta} S_{\alpha \beta}(t) x \\
= & \frac{1}{2 \pi i} \int_{\Gamma} e^{\lambda t} \lambda^{\eta+\alpha-\beta} A^{\theta}\left(\lambda^{\alpha}+A\right)^{-1} x d \lambda \\
= & \frac{1}{2 \pi i} \int_{\Gamma} e^{\lambda t} \lambda^{\eta-\beta} A^{\theta}\left(1+\lambda^{-\alpha} A\right)^{-1} x d \lambda \\
= & \frac{1}{2 \pi i} \sum_{j= \pm 1} \int_{0}^{\infty} e^{s e^{i j \rho} t} s^{\eta-\beta} e^{i j \rho(\eta-\beta)} A^{\theta}\left(1+e^{-i j \alpha \rho} s^{-\alpha} A\right)^{-1} x e^{i j \rho} d s .
\end{aligned}
$$

Continue with the change of variables $s=u^{-\frac{1}{\alpha}}, d s=-\frac{1}{\alpha} u^{-1-\frac{1}{\alpha}} d u$ and later use (3.1), (5.4) and (5.2):

$$
\begin{aligned}
& D_{t}^{\eta} A^{\theta} S_{\alpha \beta}(t) x \\
= & \frac{1}{2 \pi i} \sum_{j= \pm 1} e^{i j \rho(1+\eta-\beta)} . \\
& \int_{0}^{\infty} e^{e^{i j \rho} t u^{-1 / \alpha}} u^{(\beta-\eta) / \alpha} u^{-\theta}(u A)^{\theta}\left(1+e^{-i j \alpha \rho} u A\right)^{-1} x u^{-1 / \alpha} \frac{1}{\alpha} \frac{d u}{u} \\
= & \frac{1}{2 \pi i \alpha} \sum_{j= \pm 1} e^{i j \rho(1+\eta-\beta)} \int_{0}^{\infty} e^{e^{i j \rho} t u^{-1 / \alpha}} u^{-1 /(2 \alpha)}(u A)^{\theta}\left(1+e^{-i j \alpha \rho} u A\right)^{-1} x \frac{d u}{u} \\
= & \sum_{j= \pm 1} \int_{0}^{\infty} k_{j}(u, t) \psi_{j}(u A) x \frac{d u}{u} .
\end{aligned}
$$

By (5.5) and (5.6) we have Proposition 3.3.

\section{Proof of Theorem 3.1}

Proof. As in [7] we note first that it suffices to consider $\mathcal{F}$-adapted finite step processes $G: \mathbb{R}^{+} \times \Omega \rightarrow \mathcal{D}\left(A_{H}\right)$ with $A_{H}=A \otimes I_{H}$.

By Proposition 3.3, with $X=L_{q}(\mathcal{O} ; H)$ we have the representation

$$
A^{\theta} D_{t}^{\eta} S_{\alpha \beta}(t) x=\sum_{j= \pm 1} \int_{0}^{\infty} k_{j}(u, t)\left[\varphi_{j}(u A)\right]^{2} x \frac{d u}{u},
$$

with

$$
\varphi_{j} \in H_{0}^{\infty}\left(\Sigma_{\omega_{1}}\right), \omega_{1} \in(\omega, \pi) .
$$

(This corresponds to relation (4.2) of [7]). The functions $k_{j}$ depend on $\alpha, \beta, \eta$ and, through (3.1), on $\theta$. Below, we do not explicitly write out this dependence. 
By the stochastic Fubini theorem, and by (6.1), for $t \geq 0$,

$$
\begin{aligned}
& A^{\theta} D_{t}^{\eta} \int_{0}^{t} S_{\alpha \beta}(t-s) G(s) d W_{H}(s) \\
= & \sum_{j= \pm 1} \int_{0}^{t} \int_{0}^{\infty}\left[\varphi_{j}(u A)\right]^{2} k_{j}(u, t-s) G(s) \frac{d u}{u} d W_{H}(s) \\
= & \sum_{j= \pm 1} \int_{0}^{\infty}\left[\varphi_{j}(u A)\right]^{2}\left(I_{j}(u) G\right)(t) \frac{d u}{u},
\end{aligned}
$$

with

$$
\left(I_{j}(u) G\right)(t)=\int_{0}^{t} k_{j}(u, t-s) G(s) d W_{H}(s) .
$$

The remainder of the proof follows that of [7, Theorem 4.3].

Our notation (as in [7]) resorts to the concept of Banach function spaces. Let $E$ be a Banach function space over $(\mathcal{O}, \Sigma, \mu)$ and $\mathcal{H}$ a Hilbert space. Then $E(\mathcal{H})$ denotes the space of all strongly $\mu$ measurable functions $G: \mathcal{O} \rightarrow \mathcal{H}$, such that $\|G(\cdot)\|_{\mathcal{H}}$ belongs to $E$. In the sequel, we will use the following spaces:

$$
\begin{aligned}
E & =L_{q}(\mathcal{O} ; \mathbb{R}), \\
\mathcal{H} & =L_{2}\left(\mathbb{R}^{+} ; \frac{d u}{u}\right), \\
E_{1} & =L_{p}\left(\mathbb{R}^{+} \times \Omega ; L_{q}(\mathcal{O} ; H)\right), \\
E_{2} & =L_{p}\left(\mathbb{R}^{+} \times \Omega ; L_{q}(\mathcal{O} ; \mathbb{R})\right) .
\end{aligned}
$$

Let $p^{-1}+\left(p^{\prime}\right)^{-1}=1, q^{-1}+\left(q^{\prime}\right)^{-1}=1$. Then

$$
\begin{aligned}
E(\mathcal{H}) & =L_{q}(\mathcal{O} ; \mathcal{H})=L_{q}\left(\mathcal{O} ; L_{2}\left(\mathbb{R}^{+} ; \frac{d u}{u}\right)\right), \\
E_{2}(\mathcal{H}) & =L_{p}\left(\mathbb{R}^{+} \times \Omega ; L_{q}\left(\mathcal{O} ; L_{2}\left(\mathbb{R}^{+} ; \frac{d u}{u}\right)\right)\right), \\
E^{*} & =L_{q^{\prime}}(\mathcal{O} ; \mathbb{R}) ; \\
E_{2}^{*} & =L_{p^{\prime}}\left(\mathbb{R}^{+} \times \Omega ; L_{q^{\prime}}(\mathcal{O} ; \mathbb{R})\right) .
\end{aligned}
$$

Thus

$$
\begin{gathered}
E^{*}(\mathcal{H})=L_{q^{\prime}}\left(\mathcal{O} ; L_{2}\left(\mathbb{R}^{+} ; \frac{d u}{u}\right)\right), \\
E_{2}^{*}(\mathcal{H})=L_{p^{\prime}}\left(\mathbb{R}^{+} \times \Omega ; L_{q^{\prime}}\left(\mathcal{O} ; L_{2}\left(\mathbb{R}^{+} ; \frac{d u}{u}\right)\right)\right) .
\end{gathered}
$$


Now take any $\xi^{*} \in E_{2}^{*}$. We wish to estimate $\left\langle A^{\theta} D_{t}^{\eta} U, \xi^{*}\right\rangle_{E_{2}, E_{2}^{*}}$. One has, using Fubini,

$$
\begin{aligned}
= & \sum_{j=-1,+1} \mathcal{E}\left(\int_{0}^{\infty}<\int_{0}^{\infty}\left[\varphi_{j}(u A)\right]^{2}\left(I_{j}(u) G\right)(t) \frac{d u}{u}, \xi^{*}(t)>_{E, E^{*}} d t\right) \\
= & \sum_{j=-1,+1} \mathcal{E} \int_{0}^{\infty} \int_{\mathcal{O}} \int_{0}^{\infty}\left[\varphi_{j}(u A)\left(I_{j}(u) G\right)(t)\right](x) \\
& {\left[\varphi_{j}\left(u A^{*}\right) \xi^{*}(t)\right](x) \frac{d u}{u} d x d t . }
\end{aligned}
$$

So, using again Fubini and twice Hölders inequality,

$$
\begin{aligned}
& \left|<A^{\theta} D_{t}^{\eta} U, \xi^{*}>_{E_{2}, E_{2}^{*}}\right| \\
\leq & \sum_{j=-1,+1} \mathcal{E} \int_{0}^{\infty} \int_{\mathcal{O}}\left\|\left[\varphi_{j}(u A)\left(I_{j}(u) G\right)(t)\right](x)\right\|_{\mathcal{H}} \\
& \left.\| \varphi_{j}\left(u A^{*}\right) \xi^{*}(t)\right](x) \|_{\mathcal{H}} d x d t \\
\leq & \sum_{j= \pm 1}\left\|\varphi_{j}(u A) I_{j}(u) G\right\|_{E_{2}(\mathcal{H})}\left\|\varphi_{j}\left(u A^{*}\right) \xi^{*}\right\|_{E_{2}^{*}(\mathcal{H})} .
\end{aligned}
$$

The estimation of the last factor on the right side of (6.5) may be done by an application of the following Proposition ([7], [8]). (In [7] this proposition is stated under the assumption that the bounded functional calculus has angle $\omega<\frac{\pi}{2}$ but a simple rescaling argument shows that the proposition is true under the assumption $\omega<\pi$ ).

Proposition 6.1. Take $q \in(1, \infty)$, and assume that $A$ has a bounded $\mathbf{H}^{\infty}\left(\Sigma_{\omega}\right)$-calculus on $L_{q}(\mathcal{O} ; \mathbb{R})$ for some $\omega \in(0, \pi)$, and let $\omega_{1} \in(\omega, \pi)$ be arbitrary. Then, for each $\varphi \in H_{0}^{\infty}\left(\Sigma_{\omega_{1}}\right)$ there exists a constant $c$ such that

$$
\begin{gathered}
\|\varphi(u A) x\|_{E(\mathcal{H})} \leq c\|x\|_{L_{q}(\mathcal{O} ; \mathbb{R})}, x \in L_{q}(\mathcal{O} ; \mathbb{R}), \\
\left\|\varphi\left(u A^{*}\right) x^{*}\right\|_{E^{*}(\mathcal{H})} \leq c\left\|x^{*}\right\|_{L_{q^{\prime}}(\mathcal{O} ; \mathbb{R})}, x^{*} \in L_{q^{\prime}}(\mathcal{O} ; \mathbb{R}) .
\end{gathered}
$$

By (3.2) and (6.2) we may apply the above Proposition pointwise in $\mathbb{R}^{+} \times \Omega$. Thus, by (6.7),

$$
\left\|\varphi_{j}\left(u A^{*}\right) \xi^{*}\right\|_{E_{2}^{*}(\mathcal{H})} \leq c\left\|\xi^{*}\right\|_{E_{2}^{*}} .
$$

The estimation of the terms

$$
\left\|\varphi_{j}(u A) I_{j}(u) G\right\|_{E_{2}(\mathcal{H})}
$$

requires a more elaborate machinery.

As in [7], define ( $M$ as in (3.8))

$$
\mathcal{K}=\left\{k \in W_{1}^{1}\left(\mathbb{R}^{+}\right)\left|\int_{0}^{\infty} \sqrt{t}\right| k^{\prime}(t) \mid d t \leq M\right\},
$$


and for $k \in \mathcal{K}$, and an $\mathcal{F}$-adapted finite rank step process $G: \mathbb{R}^{+} \times \Omega \rightarrow$ $L_{q}(\mathcal{O} ; H)$, the process

$$
I(k) G(t)=\int_{0}^{t} k(t-s) G(s) d W_{H}(s), t \geq 0 .
$$

By the Ito isometry, these (scalarvalued) processes are welldefined. To extend $I(k)$ to a bounded operator from $L_{p, \mathcal{F}}\left(\mathbb{R}^{+} \times \Omega ; L_{q}(\mathcal{O} ; H)\right)$ into $L_{p}\left(\mathbb{R}^{+} \times \Omega ; L_{q}(\mathcal{O} ; \mathbb{R})\right)$, use the inequality (see $(2.1)$ of $[7]$ )

$$
\mathcal{E}\left\|\int_{0}^{\infty} G d W_{H}\right\|_{L_{q}(\mathcal{O} ; \mathbb{R})}^{p} \leq c \mathcal{E}\|G\|_{L_{2}\left(\mathbb{R}^{+} ; L_{q}(\mathcal{O} ; H)\right)}^{p} .
$$

Note that by Proposition 3.3, in particular by (3.8), the functions $k_{j} \in$ $\mathcal{K}$.

The operators $I(k)$ are uniformly bounded, by the fact that

$$
\sup _{k \in \mathcal{K}} \int_{0}^{\infty} k^{2}(t) d t<\infty
$$

To see this, write

$\int_{0}^{\infty} k^{2}(t) d t=-\int_{0}^{\infty} k^{\prime}(s) \int_{0}^{s} k(t) d t d s=-\int_{0}^{\infty} s^{\frac{1}{2}} k^{\prime}(s)\left[s^{-\frac{1}{2}} \int_{0}^{s} k(t) d t\right] d s$ and observe that

$$
\begin{aligned}
\left|s^{-\frac{1}{2}} \int_{0}^{s} k(t) d t\right| & =\left|\int_{s}^{\infty} s^{\frac{1}{2}} k^{\prime}(\tau) d \tau+\int_{0}^{s} \tau s^{-\frac{1}{2}} k^{\prime}(\tau) d \tau\right| \\
& \leq \int_{0}^{\infty} \tau^{\frac{1}{2}}\left|k^{\prime}(\tau)\right| d \tau
\end{aligned}
$$

But even more is true, the operator family $I$ is $\mathcal{R}$-bounded, [7, Theorem 3.1]:

Proposition 6.2. For all $p \in(2, \infty)$ and $q \in[2, \infty)$, the operator family $I$ is $\mathcal{R}$-bounded from $L_{p, \mathcal{F}}\left(\mathbb{R}^{+} \times \Omega ; L_{q}(\mathcal{O} ; H)\right)$ to $L_{p}\left(\mathbb{R}^{+} \times\right.$ $\left.\Omega ; L_{q}(\mathcal{O} ; \mathbb{R})\right)$. The same result is true if $p=q=2$.

For the definition of $\mathcal{R}$-boundedness and the proof of this proposition, see [7].

The following multiplier result makes use of the $\mathcal{R}$-boundedness. (See [7], [8].)

Proposition 6.3. Let $E_{1}, E_{2}$ be Banach function spaces with finite cotype and let $\nu$ be a $\sigma$-finite Borel measure on $\mathbb{R}^{+}$. Let $M: \mathbb{R}^{+} \rightarrow$ $\mathcal{L}\left(E_{1}, E_{2}\right)$ be a function with the following properties:

(1) for all $x \in E_{1}$ the function $t \rightarrow M(t) x$ is strongly $\nu$-measurable in $E_{2}$,

(2) the range $\mathcal{M}=\left\{M(t) \mid t \in \mathbb{R}^{+}\right\}$is $\mathcal{R}$-bounded in $\mathcal{L}\left(E_{1}, E_{2}\right)$.

Then, for $G \in E_{1}\left(L_{2}\left(\mathbb{R}^{+} ; \nu\right)\right)$ we have $M G \in E_{2}\left(L_{2}\left(\mathbb{R}^{+} ; \nu\right)\right)$ and

$$
\|M G\|_{E_{2}\left(L_{2}\left(\mathbb{R}^{+}, \nu\right)\right)} \leq \mathcal{R}(\mathcal{M})\|G\|_{E_{1}\left(L_{2}\left(\mathbb{R}^{+} ; \nu\right)\right)} .
$$


Above, $\mathcal{R}(\mathcal{M})$ denotes the $\mathcal{R}$-bound of the operator family $\mathcal{M}$.

Take $E_{1}, E_{2}$ as in $(6.3)$ and recall that $f \in E_{1}(\mathcal{H})$ is defined by requiring $\|f\|_{\mathcal{H}} \in E_{1}$. Then note that

$$
\varphi_{j}(u A) I_{j}(u) G=I_{j}(u) \varphi_{j}\left(u A_{H}\right) G,
$$

apply Proposition 6.3 and (6.6) of Proposition 6.1 pointwise in $\mathbb{R}^{+} \times \Omega$ to obtain

$$
\begin{aligned}
& \left\|\varphi_{j}(u A) I_{j}(u) G\right\|_{E_{2}(\mathcal{H})} \leq \\
& \mathcal{R}\left(I_{j}(u), u \in \mathbb{R}^{+}, j= \pm 1\right)\left\|\varphi_{j}\left(u A_{H}\right) G\right\|_{E_{1}(\mathcal{H})} \leq \\
& c \mathcal{R}\left(I_{j}(u), u \in \mathbb{R}^{+}, j= \pm 1\right)\|G\|_{E_{1}} .
\end{aligned}
$$

By (3.8) and by Proposition 6.2,

$$
\mathcal{R}\left(I_{j}(u), u \in \mathbb{R}^{+}, j= \pm 1\right) \leq c<\infty .
$$

By (6.8), (6.9), (6.10),

$$
\left|<A^{\theta} D_{t}^{\eta} U, \xi^{*}>_{E_{2}, E_{2}^{*}}\right| \leq c\|G\|_{E_{1}}\left\|\xi^{*}\right\|_{E_{2}^{*}} .
$$

Taking the supremum over $\left\|\xi^{*}\right\|_{E_{2}^{*}} \leq 1$ gives

$$
\left\|A^{\theta} D_{t}^{\eta} U\right\|_{E_{2}} \leq c\|G\|_{E_{1}} .
$$

Recall the definitions of $E_{1}$ and $E_{2}$ to see that (3.3) has been obtained.

Acknowledgement - We thank Jan van Neerven, Mark Veraar and Lutz Weis for their inspiring paper and encouraging comments.

\section{REFERENCES}

[1] C. Martinez Carracedo and M. Sanz Alix, The Theory of Fractional Powers of Operators. North Holland Mathematics Studies 187, North Holland, Amsterdam, 2001.

[2] Ph. Clement, S-O. Londen and G. Simonett, Quasilinear evolutionary equations and continuous interpolation spaces, J. Differential Eqs., 196 (2004), 418-447.

[3] W. Desch and S-O. Londen, A generalization of an inequality by N.V. Krylov, J. Evolution Eqs., 9 (2009), 525-560.

[4] W. Desch and S-O. Londen, An $L_{p}$-theory for stochastic integral equations. To appear, J. Evolution Eqs.

[5] N.V. Krylov, A parabolic Littlewood-Paley inequality with applications to parabolic equations, Topological Methods in Nonlinear Analysis, Journal of the Juliusz Schauder Center 4 (1994), 355-364.

[6] N.V. Krylov, An analytic approach to SPDEs. In Stochastic Partial Differential Equations: Six Perspectives, R.A. Carmona and B. Rozovskii, eds., A.M.S. Mathematical Surveys and Monographs, 64 (1999), 185-242.

[7] J. van Neerven, M. Veraar and L. Weis, Stochastic maximal $L_{p}$-regularity. Preprint 2010.

[8] L.W. Weis, The $H^{\infty}$ holomorphic functional calculus for sectorial operators - a survey. In Partial Differential Equations and Functional Analysis, volume 168 of Oper. Theory Adv.Appl., pp. 263-294. Birkhäuser, Basel, 2006. 
[9] R. Zacher, Quasilinear Parabolic Problems with Nonlinear Boundary Conditions, Dissertation, Martin-Luther-Universität Halle-Wittenberg, 2003. 


\section{Recent reports at the department of Mathematics and Systems Analysis}

Stig-Olof Londen, Hana Petzeltovà

Regularity and separation from potential barriers for a non-local phase-field system

Helsinki University of Technology Institute of Mathematics Research Reports A598, December 2010

Mikko Byckling

Preconditioning for standard and two-sided Krylov subspace methods

Helsinki University of Technology Institute of Mathematics Research Reports A597, December 2010

Kimmo Berg

A Learning Approach for Nonlinear Pricing Problem

Systems Analysis Laboratory Research Reports A105, December 2010

Olavi Nevanlinna

Multicentric Holomorphic Calculus

Helsinki University of Technology Institute of Mathematics Research Reports A596, November 2010

Juho Könnö, Rolf Stenberg

Numerical computations with $\mathrm{H}$ (div)-finite elements for the Brinkman problem

Helsinki University of Technology Institute of Mathematics Research Reports A595, November 2010

Atte Aalto, Jarmo Malinen

Cauchy problems from networks of passive boundary control systems

Helsinki University of Technology Institute of Mathematics Research Reports A594, October 2010

Toni Lassila

Model reduction and level set methods for shape optimization problems

Helsinki University of Technology Institute of Mathematics Research Reports A593, October 2010

Olavi Nevanlinna

Upper bounds for R-linear resolvents

Helsinki University of Technology Institute of Mathematics Research Reports A592, September 2010

Juhana Siljander

Regularity for degenerate nonlinear parabolic partial differential equations

Helsinki University of Technology Institute of Mathematics Research Reports A591, September 2010

\section{Ehsan Azmoodeh}

Riemann-Stieltjes integrals with respect to fractional Brownian motion and applications

Helsinki University of Technology Institute of Mathematics Research Reports A590, September 2010

Antti Rasila, Jarno Talponen

Convexity properties of quasihyperbolic balls on Banach spaces

Helsinki University of Technology Institute of Mathematics Research Reports A589, August 2010

Ville Brummer

Participatory Approaches to Foresight and Priority-Setting in Innovation Networks

Systems Analysis Laboratory Research Reports A104, June 2010

Kalle Mikkola

Real solutions to control, approximation, factorization, representation, Hankel and Toeplitz problems Helsinki University of Technology Institute of Mathematics Research Reports A588, June 2010 
ISBN: 978-952-60-4021-9 (pdf)

ISBN:

ISSN-L: 1799-4896

ISSN: $1799-490 X$ (pdf)

ISSN: $1799-4896$

Aalto University

School of Science

Department of Mathematics and Systems Analysis aalto.fi

BUSINESS +

ECONOMY

ART +

DESIGN +

ARCHITECTURE

SCIENCE +

TECHNOLOGY

CROSSOVER

DOCTORAL

DISSERTATIONS 